\title{
DINÂMICA ECONÔMICA DAS FLUTUAÇÕES NA PRODUÇÃO DE CANA-DE-AÇÚCAR
}

\author{
Luiz Fernando Satolo* \\ Mirian Rumenos Piedade Bacchi ${ }^{\dagger}$
}

\begin{abstract}
Resumo
O objetivo deste trabalho é avaliar o papel de choques de oferta e de demanda na evolução recente da produção de cana-de-açúcar. Os testes de raiz unitária foram feitos utilizando a metodologia DF-GLS (Elliot et al., 1992) e os de cointegração, a de Johansen (1988). O modelo estimado foi um VEC - Modelo de Auto-Regressão Vetorial com Correção de Erro, sendo a identificação feita pelo procedimento de Bernanke- Sims. Os resultados indicam que os choques de oferta têm impacto acumulado permanente sobre a produção de cana-de-açúcar, enquanto choques de demanda apresentaram efeitos temporários. Constatou-se que os estímulos advindos da oferta foram os mais importantes para explicar as flutuações na produção de cana-de-açúcar.
\end{abstract}

Palavras-chave: Agroindústria canavieira, Cana-de-açúcar, Séries temporais.

\begin{abstract}
The objective of this paper is to evaluate the whole of shocks in supply and in demand on recent developments in sugar cane production. Unit root tests were performed following DF-GLS (Elliot et al. 1992) methodology and co-integration tests, used Johansen's (1988). The model was estimated as a structural Vector Error Correction, with innovations calculated through the Bernanke-Sims decomposition. Supply shocks had a permanent impact over cane production, but demand shocks had a transitory effect over it. Innovations coming from the supply side were the most important in explaining the fluctuations in the sugar cane production in Brazil.
\end{abstract}

Keywords: Sugar and ethanol sector, Sugar cane, Time series.

JEL classification: Q11, C32.

\footnotetext{
* Doutorando em Economia Aplicada pela ESALQ/USP. E-mail: luizsatolo@hotmail.com. O autor agradece o apoio financeiro do $\mathrm{CNPq}$.

† Professora do Departamento de Economia, Administração e Sociologia, ESALQ/USP. Endereço: Av. Pádua Dias, 11, Cx.Postal 9, CEP 13418-900, Piracicaba, São Paulo, Brasil. E-mail: mrpbacch@esalq.usp.br.
} 


\section{Introdução}

Recentes acontecimentos atraíram a atenção do mundo para a cadeia produtiva de açúcar e álcool no Brasil, deflagrando uma corrida de investimentos nesse setor. Em 2006, mais de 350 unidades industriais (usinas e destilarias) estavam em operação no Brasil - e outras 126 já estavam em fase de projeto/construção. Essa nova onda de expansão do setor sucroalcooleiro vem acompanhada de um aumento no emprego e na renda. Estima-se que, em 2006, cerca de 4,6 milhões de trabalhadores estivessem direta ou indiretamente ligados ao setor sucroalcooleiro (Mariante 2007).

Segundo a Confederação da Agricultura e Pecuária do Brasil - CNA (2008) CNA (2008), no referido ano, o Valor Bruto da Produção - VBP do setor de cana-de-açúcar atingiu R $\$ 19,25$ bilhões - representando quase $18 \%$ do VBP da agricultura nacional, que somou $\mathrm{R} \$ 107,62$ bilhões. As exportações de açúcar e álcool, em 2006, totalizaram US\$ 6,9 bilhões - ou seja, mais de 15\% do total exportado pelo agronegócio brasileiro, cujo valor estimado foi $\mathrm{R} \$ 45,3$ bilhões.

A expectativa de agentes do setor é de que as exportações brasileiras continuem crescendo. Tal expectativa está fundamentada não apenas na derrota da União Européia - UE no painel aberto pelo Brasil junto à Organização Mundial do Comércio - OMC para analisar os subsídios concedidos à produção de açúcar, mas também na demanda mundial crescente por energia limpa e renovável. Além disso, deve-se destacar que está também em curso uma notória expansão do mercado doméstico: programas de distribuição de renda (como o Bolsa Escola e o Bolsa Família, por exemplo) aumentaram a renda disponível para consumo nas classes sociais mais baixas, enquanto facilidades no acesso ao crédito contribuíram para um aumento crescente na venda de veículos bicombustíveis.

O Brasil destaca-se nos mercados de açúcar e de álcool entre os principais países produtores, consumidores e exportadores por causa de sua competitividade na produção de cana-de-açúcar. Ao longo da história recente, entretanto, esse argumento nem sempre foi válido: a produtividade da cana-de-açúcar no Brasil cresceu significativamente a partir da segunda metade da década de 70, chegando a ultrapassar a média mundial apenas nos anos 80 . Esse aumento da produtividade média dos canaviais brasileiros pode ser atribuído à crescente participação da região CS - com destaque para o Estado de São Paulo na produção nacional.

Como se sabe, o crescimento da produção - fundamentado em ganhos de produtividade - não ocorre de forma sustentada sem uma expansão do mercado: o aumento da demanda (interna ou externa) simultaneamente ao avanço tecnológico é condição necessária para que se evite uma queda acentuada nos preços, permitindo a difusão de novas tecnologias. Por outro lado, um crescimento da produção sem avanço tecnológico (ou seja, sem ganhos de produtividade) também está ligado a uma expansão do mercado consumidor: havendo crescimento da demanda, o nível de preços aumenta - permitindo que novos produtores entrem no mercado a custos de oportunidade crescentes. Mas, nesse caso, o crescimento da produção ocorre a preços crescentes (Alves 2006).

No caso da produção de cana-de-açúcar no Brasil, quais estímulos predominaram: os da oferta ou os da demanda?

Ao longo desse trabalho, busca-se responder a essa indagação. Fazendo uso da metodologia VEC estrutural, são estimados os impactos de choques 
nas variáveis do modelo e a decomposição da variância dos erros de previsão. Estabelecendo-se os impactos, as funções de resposta a impulso (que exibem o comportamento das variáveis ao longo do tempo após a ocorrência do choque) poderão ser analisadas. Em conjunto com a decomposição da variância do erro de previsão (que permite avaliar o poder explicativo de cada variável sobre as demais), é possível identificar qual tem sido a dinâmica econômica das flutuações na produção de cana-de-açúcar.

Devido à disponibilidade de dados e às grandes disparidades regionais na produção de cana, a análise estará restrita ao Estado de São Paulo. Contudo, como destaca da Costa (2000, p. 5): "a relevância de estudar o setor sucroalcooleiro de São Paulo tem residido na representatividade do estado na produção, tanto no contexto do mercado doméstico como do mercado internacional". Entre as safras 1990/91 e 2006/07, São Paulo foi responsável, em média, por quase $75 \%$ da produção do setor sucroalcooleiro na região CS equivalente a mais $60 \%$ das produções de cana, açúcar e álcool no Brasil.

\section{Modelos para a decomposição das flutuações do produto}

Em um estudo pioneiro, Blanchard e Quah (1989) propuseram que o Produto Nacional Bruto norte-americano é afetado por mais de um tipo de distúrbio: choques de demanda e de oferta - que teriam, respectivamente, um efeito temporário e permanente sobre a produção. Essa classificação dos choques está fundamentada no ponto de vista Keynesiano de flutuações: devido à rigidez nominal, que não permite a alteração dos preços (por causa dos custos de ajustamento) nem dos salários (por causa da existência de contratos), as empresas estão dispostas a manter constantes seus preços relativos face a um choque de demanda - provocando apenas variações de curto-prazo na produção; já os choques de oferta, por afetarem a parte real da economia, apresentariam também efeito no longo-prazo.

Os autores definem como choques temporários aqueles cujo efeito atinge o pico depois de 1 ano, desaparecendo no segundo ou terceiro ano (efeitos positivos somam-se a negativos, desaparecendo quando considerados de forma acumulada). Já os choques permanentes são aqueles que aumentam constantemente ao longo de 2 anos, atingindo um platô no quinto ano - de forma acumulada eles perduram no tempo. Os seguintes pressupostos foram adotados para a definição de um modelo capaz de decompor as variações do produto em choques de demanda e de oferta:

- Choques temporários (de demanda) não afetam a produção no longoprazo;

- Choques permanentes (de oferta) podem afetar a produção no longoprazo;

- Os distúrbios são não-correlacionados;

- Cada um desses componentes pode ser representado, separadamente, como um termo de defasagens distribuídas invertível.

No Brasil, dois estudos recentes adaptaram a representação de choques de oferta e demanda proposta por Blanchard e Quah (1989) para a decomposição das variações na produção agrícola. Barros et al. (2006) e Alves et al. (2008) 
analisaram, respectivamente, o padrão de crescimento da agricultura e da cotonicultura brasileiras.

Barros et al. (2006) consideram que a demanda por produtos agrícolas brasileiros divide-se em duas: doméstica e externa. A demanda doméstica foi representada pela diferença entre a renda real e os preços relativos da agricultura e a demanda externa, pela taxa de câmbio. A oferta de produtos agrícolas foi representada pela multiplicação da produtividade da terra com a área total plantada. Pressupôs-se que a decisão com relação à área a ser plantada é tomada com base na expectativa de preços para o período.

Alves et al. (2008) utilizaram uma representação semelhante. A exportação de algodão, entretanto, foi representada pelo excedente ofertado no mercado interno. O modelo econômico assumiu que os preços interno e externo apresentam evolução semelhante no longo-prazo, apesar de admitir-se um período de ajustamento entre os mesmos. Características intrínsecas ao mercado de algodão permitiram que o preço interno fosse considerado exógeno.

\section{Modelo econômico}

O modelo aqui apresentado é uma versão adaptada dos modelos utilizados em Alves et al. (2008) e Barros et al. (2006). A equação de demanda pode ser expressa como:

$$
y_{t}^{d}=m_{t}-p_{t}^{d}+v_{t}^{d}
$$

onde $y^{d}$ é a quantidade domesticamente demandada de cana-de-açúcar, $m$ é a renda nacional real e $p^{d}$ é um índice que representa os preços dos produtos finais (açúcar e álcool) praticados no mercado doméstico, com o resíduo $\left(v_{t}^{d}\right)$ representando outras variáveis que também afetam a demanda. Já a oferta pode ser expressa por:

$$
y_{t}^{s}=n_{t}+\theta_{t}+v_{t}^{s}
$$

onde $y^{s}$ é a quantidade ofertada de cana-de-açúcar, $n$ é a área plantada e $\theta$ é a produtividade da terra. O termo de resíduo, $v_{t}^{s}$, representa outras variáveis que também afetam a oferta. Como a cana-de-açúcar é um produto altamente perecível, não permitindo a formação de estoques, a quantidade ofertada em determinado ano é idêntica à quantidade produzida.

Embora a cana-de-açúcar não seja exportada in natura, o açúcar e o álcool são importantes itens da pauta de exportações brasileiras. Por isso, a quantidade de cana produzida e destinada ao mercado externo $(x)$ será aqui representada por:

$$
x_{t}=y_{t}^{s}-y_{t}^{d}
$$

A pressuposição de que as exportações de açúcar dependem da oferta e demanda domésticas não implica assumir que elas não recebam influência de preço internacional e do câmbio. A oferta depende do preço no mercado interno e este, por sua vez, depende grandemente do preço do produto no mercado internacional e do câmbio. Estudos sobre o processo de formação de preço de açúcar no mercado doméstico mostram que o preço internacional e o câmbio explicam grande parte das variações dos preços internos (Barros et al. 2006, Bacchi 2009).

Além dessas equações, para que o modelo econométrico possa ser estimado, fazem-se necessárias algumas pressuposições sobre o processo gerador 
das séries que se encontram no lado direito das equações (1) e (2). São considerados os seguintes choques:

$$
\begin{gathered}
m_{t}=m_{t-1}+e_{t}^{m} \\
p_{t}^{d}=p_{t-1}^{d}+e_{t}^{d}+e_{t}^{s} \\
p_{t}^{s}=p_{t-1}^{s}+e_{t}^{s}+e_{t}^{d} \\
\theta_{t}=\theta_{t-1}+e_{t}^{\theta}+e_{t}^{s} \\
n_{t}=\mathrm{E}\left(p_{t}^{s}\right)+e_{t}^{n}
\end{gathered}
$$

sendo: $e^{m}$ choque de renda interna; $e^{d}$ choque de preço doméstico proveniente da demanda (via preços do açúcar e do álcool); $e^{s}$ choque de preço doméstico proveniente da oferta (via preço da cana-de-açúcar); $e^{\theta}$ choque de produtividade; $e^{n}$ choque de área plantada. Com

$$
e_{t}^{n}=e_{t-1}^{n}+u_{t}
$$

Por hipótese, considera-se que todos os choques são não-correlacionados. Dessa forma, deve-se entender os choques de preço da cana $\left(e^{s}\right)$ sobre os preços de açúcar e álcool $\left(p^{d}\right)$ como variações inesperadas no preço relativo entre matéria-prima e produtos finais. Analogamente, choques de preços de açúcar e álcool $\left(e^{d}\right)$ sobre o preço da cana $\left(p^{s}\right)$ também representam essas variações no preço relativo. Por outro lado, deve-se entender choques de produtividade $\left(e^{\theta}\right)$ como variações na produtividade da terra $(\theta)$ causadas por motivos alheios ao preço da cana - como fatores climáticos, por exemplo. As mudanças no rendimento da lavoura provenientes da adoção de novas práticas de manejo quando os preços da cana variam são representadas pelos choques $e^{s}$ na equação (7).

Além disso, exceto por $e^{n}$, os choques têm médias zero e não apresentam autocorrelações. Os choques de área plantada são por hipótese autocorrelacionados porque, apesar da influência que os preços sobre a área cultivada, a cana-de-açúcar é uma cultura semi-perene. A rigidez que caracteriza os investimentos realizados (ou não) em canaviais implica em uma interdependência temporal dos choques de área. Já $u_{t}$ possui as mesmas propriedades que os demais choques, isto é, média zero e ausência de autocorrelação.

\subsection{A taxa de crescimento das variáveis}

Nesta seção, são apresentadas as expressões que descrevem a taxa de crescimento das variáveis incluídas no modelo. A taxa de crescimento da oferta de cana-de-açúcar pode ser expressa como:

$$
\Delta y_{t}^{S}=e_{t-1}^{s}+e_{t-1}^{d}+u_{t}+e_{t}^{\theta}+e_{t}^{s} .
$$

Ou seja, aumentos de preço em um período alteram a expectativa de preços e, por isso, elevam tanto a área quanto a produção no período seguinte. Já aumentos de área (per se), de produtividade e de preço da cana favorecem o aumento da oferta contemporaneamente. 
As taxas de crescimento da demanda e da área plantada podem ser escritas, respectivamente, como:

$$
\begin{gathered}
\Delta y_{t}^{d}=e_{t}^{m}-e_{t}^{d}-e_{t}^{s} \\
\Delta n_{t}=e_{t-1}^{s}+e_{t-1}^{d}+u_{t}
\end{gathered}
$$

No caso das exportações tem-se que:

$$
\Delta x_{t}=e_{t-1}^{s}+e_{t-1}^{d}+u_{t}+e_{t}^{\theta}-e_{t}^{m}+e_{t}^{d}+2 e_{t}^{s} .
$$

Portanto, espera-se que choques contemporâneos de oferta (área e produtividade) e de preços domésticos (tanto de cana quanto de açúcar e álcool) afetem positivamente as exportações do setor sucroalcooleiro, enquanto choques contemporâneos de renda doméstica tendam a diminuí-las. Choques defasados dos preços também aumentam as exportações: com o aumento do nível de preços, o consumo doméstico é diminuído e - para se evitar um aumento excessivo de estoques - grande parte do excedente doméstico acaba sendo exportada.

Com relação às taxas de crescimento das demais variáveis - renda, índice de preços de açúcar e álcool, preço da cana e produtividade - tem-se, respectivamente:

$$
\begin{gathered}
\Delta m_{t}=e_{t}^{m} \\
\Delta p_{t}^{d}=e_{t}^{d}+e_{t}^{s} \\
\Delta p_{t}^{s}=e_{t}^{s}+e_{t}^{d} \\
\Delta \theta_{t}=e_{t}^{\theta}+e_{t}^{s}
\end{gathered}
$$

\subsection{Definição da matriz de relações contemporâneas}

A forma da matriz de relações contemporâneas é de suma importância para a decomposição da variância e a determinação dos impactos em um conjunto de variáveis - os quais, neste estudo, serão utilizados para uma melhor compreensão da dinâmica econômica das flutuações na produção de cana. Por essa razão, a forma pela qual essa matriz foi extraída do modelo econômico será aqui explicitada.

A equação (13) evidencia que o modelo econométrico a ser estimado deve possuir, na verdade, seis variáveis, a saber: quantum exportado, preço da cana, índice de preços do açúcar e do álcool, área plantada, produtividade da terra e renda doméstica. A variação do quantum exportado depende da variação contemporânea das outras cinco variáveis e, por isso, essa variável é considerada endógena.

O índice de preços do açúcar e do álcool, o preço da cana e a produtividade também são variáveis endógenas - ver equações (15), (16) e (17). No caso dos preços, $p^{s}$ e $p^{d}$, endogeneidade decorre da existência de uma relação contemporânea bi-causal entre os mesmos. Com relação à produtividade, essa condição resulta do efeito contemporâneo de choques no preço da cana sobre a variável. 
De acordo com as equações (12) e (14), somente a área e a renda doméstica são variáveis exógenas: suas variações dependem contemporaneamente apenas dos choques ocorridos na própria variável.

Por tudo o que foi exposto, a matriz de relações contemporâneas pode ser escrita como:

$$
A_{0}=\left[\begin{array}{llllll}
1 & 0 & 0 & 0 & 0 & 0 \\
0 & 1 & 1 & 0 & 0 & 0 \\
0 & 0 & 1 & 1 & 0 & 0 \\
0 & 0 & 1 & 1 & 0 & 0 \\
1 & 1 & 1 & 1 & 1 & 1 \\
0 & 0 & 0 & 0 & 0 & 1
\end{array}\right]
$$

para a seguinte seqüência de variáveis: área plantada $(n)$, produtividade da terra $(\theta)$, preço da cana $\left(p^{s}\right)$, índice de preços do açúcar e do álcool $\left(p^{d}\right)$, quantum exportado $(x)$ e renda doméstica $(m)$.

Embora essa representação não evidencie a relação da produção com as demais variáveis, pode-se demonstrar que os resultados da estimativa do modelo proposto são equivalentes aos da estimativa de um modelo alternativo no qual as séries de área e produtividade são substituídas pela série de produção. ${ }^{1}$ Entretanto, nessa forma alternativa, não se consegue isolar o efeito dos choques de área e produtividade sobre a produção ou as demais variáveis do sistema - implicando a perda de informações de suma importância para explicar a dinâmica das flutuações na produção canavieira. Por isso, optou-se pela estimativa do modelo com as séries de área e produtividade - aplicando-se, posteriormente, as transformações necessárias para avaliar o comportamento da produção em reposta aos choques.

\subsection{Impacto dos choques sobre as variáveis endógenas}

Com as derivações apresentadas nas seções anteriores é possível definir, a priori, os impactos esperados de cada choque considerado sobre as variáveis endógenas do modelo.

Na Tabela 1, são apresentados os impactos correspondentes aos choques definidos no item 4.1 sobre as variáveis endógenas. O momento de intervenção é sempre o período 1 e o choque apresenta impacto contemporâneo sobre a produção, a exportação, os preços ou a produtividade se o mesmo apresentar o subscrito $t$ nas equações (10), (13), (15), (16) e (17), respectivamente. Nos casos em que o choque é defasado, seu impacto é sentido apenas no período seguinte.

Espera-se, portanto, que choques de renda doméstica não tenham impacto sobre a produção de cana-de-açúcar - uma vez que essa variável não entra na equação de oferta, afetando apenas a demanda pelo produto (e, consequentemente, as exportações). Choques de área e de produtividade devem apresentar impactos contemporâneos sobre a produção e a exportação.

Com relação a choques no preço da cana e no preço médio de açúcar e álcool, seus impactos sobre produção devem ser observados tanto contemporaneamente quanto com defasagem - exceção feita ao efeito do choque no preço

\footnotetext{
${ }^{1}$ Como $\Delta y_{t}^{s}=e_{t-1}^{s}+e_{t-1}^{d}+u_{t}+e_{t}^{\theta}+e_{t}^{s}$, onde $\Delta n_{t}=e_{t-1}^{s}+e_{t-1}^{d}+u_{t}$ e $\Delta \theta_{t}=e_{t}^{\theta}+e_{t}^{s}$, pode-se dizer que $\Delta y_{t}^{s}=\Delta n_{t}+\Delta \theta_{t}$. Ou seja, a variação da produção pode ser representada pela soma das variações da área e da produtividade em determinado ano. Dessa forma, conhecendo as respostas de $n_{t}$ e $\theta_{t}$ aos choques considerados, pode-se estimar a resposta da produção a tais inovações acumulandose seus impactos sobre a área e a produtividade.
} 
Tabela 1: Impacto esperado dos choques

\begin{tabular}{|c|c|c|c|c|c|}
\hline $\begin{array}{ll}\mathrm{Cl} \\
\mathrm{de}\end{array}$ & $\begin{array}{l}\text { oque } \\
\text { sobre }\end{array}$ & $t=0$ & $t=1$ & $t=2$ & $t=3$ \\
\hline$n$ & $y^{s}$ & $y_{0}^{S}$ & $y_{1}^{s}=y_{0}^{s}+u_{1}$ & $y_{2}^{s}=y_{1}^{s}$ & $y_{t}^{S}=y_{1}^{S}$ \\
\hline$\theta$ & $y^{s}$ & $y_{0}^{s}$ & $y_{1}^{s}=y_{0}^{s}+e_{1}^{\theta}$ & $y_{2}^{s}=y_{1}^{s}$ & $y_{t}^{s}=y_{1}^{s}$ \\
\hline$p^{s}$ & $y^{s}$ & $y_{0}^{s}$ & $y_{1}^{s}=y_{0}^{s}+e_{1}^{s}$ & $y_{2}^{s}=y_{1}^{s}+e_{1}^{s}$ & $y_{t}^{s}=y_{2}^{s}$ \\
\hline$p^{d}$ & $y^{s}$ & $y_{0}^{s}$ & $y_{1}^{s}=y_{0}^{s}$ & $y_{2}^{s}=y_{0}^{s}+e_{1}^{d}$ & $y_{t}^{s}=y_{2}^{s}$ \\
\hline$n$ & $x$ & $x_{0}$ & $x_{1}=x_{0}+u_{1}$ & $x_{2}=x_{1}$ & $x_{t}=x_{1}$ \\
\hline$\theta$ & $x$ & $x_{0}$ & $x_{1}=x_{0}+e_{1}^{\theta}$ & $x_{2}=x_{1}$ & $x_{t}=x_{1}$ \\
\hline$p^{s}$ & $x$ & $x_{0}$ & $x_{1}=x_{0}+2 e_{1}^{s}$ & $x_{2}=x_{1}+e_{1}^{s}$ & $x_{t}=x_{2}$ \\
\hline$p^{d}$ & $x$ & $x_{0}$ & $x_{1}=x_{0}+e_{1}^{d^{1}}$ & $x_{2}=x_{1}+e_{1}^{d}$ & $x_{t}=x_{2}$ \\
\hline$m$ & $x$ & $x_{0}$ & $x_{1}=x_{0}-e_{1}^{m}$ & $x_{2}=x_{1}$ & $x_{t}=x_{1}$ \\
\hline$p^{s}$ & $p^{d}$ & $p_{0}^{d}$ & $p_{1}^{d}=p_{0}^{d}+e_{1}^{s}$ & $p_{2}^{d}=p_{1}^{d}$ & $p_{t}^{d}=p_{1}^{d}$ \\
\hline$p^{d}$ & $p^{s}$ & $p_{0}^{s}$ & $p_{1}^{s}=p_{0}^{s}+e_{1}^{d}$ & $p_{2}^{s}=p_{1}^{s}$ & $p_{t}^{s}=p_{1}^{s}$ \\
\hline$p^{s}$ & $\theta$ & $\theta_{0}$ & $\theta_{1}=\theta_{0}+e_{1}^{s}$ & $\theta_{2}=\theta_{1}$ & $\theta_{t}=\theta_{1}$ \\
\hline
\end{tabular}

de açúcar e álcool sobre a produção, que não deve ser sentido contemporaneamente. Além disso, espera-se que choques no preço da cana tenham impacto contemporâneo sobre o preço de açúcar e álcool e vice-versa. O modelo também permite que choques no preço da cana afetem contemporaneamente a produtividade da lavoura, conforme exposto anteriormente.

Um aspecto importante a ser ressaltado é que, de acordo com o modelo proposto, todos os choques são permanentes. Uma vez ocorrida a variação inesperada, as variáveis endógenas atingem um novo patamar e nele permanecem até que uma nova alteração ocorra.

\section{Metodologia}

O teste DF-GLS, apresentado em Elliot et al. (1992), foi utilizado para verificar a existência de raiz unitária nas séries. Essa metodologia, uma versão eficiente do teste de Dickey-Fuller Aumentado - ADF (1987), consiste na aplicação do teste ADF à série previamente filtrada de seus componentes determinísticos. O número de defasagens utilizado no teste de raiz unitária foi determinado através do Critério de Informação de Akaike Modificado - MAIC.

A metodologia utilizada para o teste de cointegração foi a proposta por Johansen (1988), que contempla tanto a existência de mais de um vetor de cointegração quanto a endogeneidade de regressores. Esse teste consiste na estimativa de um sistema que contém exatamente $h$ relações de cointegração através do método de Máxima Verossimilhança de Informação Plena - MVIP.

O modelo econômico apresentado na seção anterior foi estimado através da metodologia de Auto-Regressão Vetorial - VAR. ${ }^{2}$ Como a decomposição de Choleski utilizada na metodologia VAR para a identificação do modelo não tem, muitas vezes, aderência com as estrutura das relações contemporâneas entre as variáveis, optou-se pela utilização da decomposição de Bernanke-

\footnotetext{
${ }^{2}$ Quando as séries apresentam-se integradas e cointegradas, o modelo deve ser ajustado com as séries nas diferenças, incluindo termo(s) de correção de erro. Nesse caso tem-se um VEC Modelo de Auto-regressão Vetorial com correção de Erro.
} 
Sims (abordagem também conhecida como VAR estrutural), na qual o modelo econômico utilizado para estabelecer essas relações (Lütkepohl 1991)

\subsection{Fonte dos dados}

Para avaliar o impacto de choques de oferta e de demanda sobre a produção de cana-de-açúcar no Estado de São Paulo foram utilizados dados anuais, abrangendo o período de 1976 a 2006. As séries de preços e de renda foram deflacionadas pelo IGP-DI da Fundação Getúlio Vargas.

A série de produção de cana-de-açúcar, em toneladas, utilizada foi a divulgada pela UNICA. A área plantada no Estado de São Paulo, em hectares, foi obtida no IBGE e complementada com os dados do Anuário Estatístico do Estado de São Paulo e dos Boletins de Safra do IAA. Já a produtividade agrícola, em $t / h a$, foi estimada com a divisão da produção pela área plantada.

Representou-se o preço da cana-de-açúcar pela série obtida junto UNICA, completada com os dados da UDOP para o período em que o método CONSECANA já estava em vigor. O índice que representa o nível de preços domésticos dos produtos finais foi calculado com a média dos preços de açúcar e álcool praticados no mercado interno, ponderados pelas respectivas quantidades produzidas (em cana equivalente). ${ }^{3}$

Os preços domésticos de açúcar e álcool foram representados pelas médias anuais dos preços mensais divulgados pela UNICA, e - dada a disponibilidade de dados - as séries de produção brasileira de açúcar, de álcool anidro e de álcool hidratado foram utilizadas nos cálculos. Essas séries também são divulgadas pela UNICA e foram completadas com os dados publicados em Szmrecsányi (1979) e Marjotta-Maistro (2002), no caso do açúcar, e em Alves (2002), no caso dos dois tipos de álcool.

A série de exportação foi representada pela quantidade, em cana equivalente, de açúcar e álcool exportada durante o período analisado. As exportações de açúcar e álcool foram recuperadas através do Sistema de Análise das Informações de Comércio Exterior - ALICEWEB para os anos subsequentes a 1989. As séries referentes à exportação de açúcar foram completadas com dados do Instituto de Pesquisa Econômica Aplicada - IPEA. No caso do álcool, entretanto, não foram encontrados dados referentes aos anos anteriores.

A renda doméstica foi representada pelo índice de salário médio na indústria de São Paulo - construído a partir da série de variação anual do referido salário real, publicada pelo IPEA.

\section{Resultados e discussão}

A seguir, são apresentadas as séries utilizadas na estimativa do modelo. Na Figura 1, encontram-se as séries de produção $\left(y^{s}\right)$ e de exportação $(x)$. Enquanto a primeira série apresentou uma tendência crescente ao longo de quase todo o período em análise, a segunda se mostrou relativamente estável até 1994 e crescente a partir desse ano. Na Figura 2, encontram-se duas das três séries que, por hipótese, geraram os choques de oferta: área plantada $(n)$ e produtividade $(\theta)$. Já na Figura 3, são ilustradas as séries que originaram os choques

\footnotetext{
${ }^{3}$ Os coeficientes utilizados para calcular a quantidade equivalente de cana-de-açúcar foram os mesmos utilizados pelo sistema CONSECANA para calcular o preço da cana: Açúcar: 1,0495; Álcool hidratado: 1,7409; Álcool anidro: 1,8169.
} 


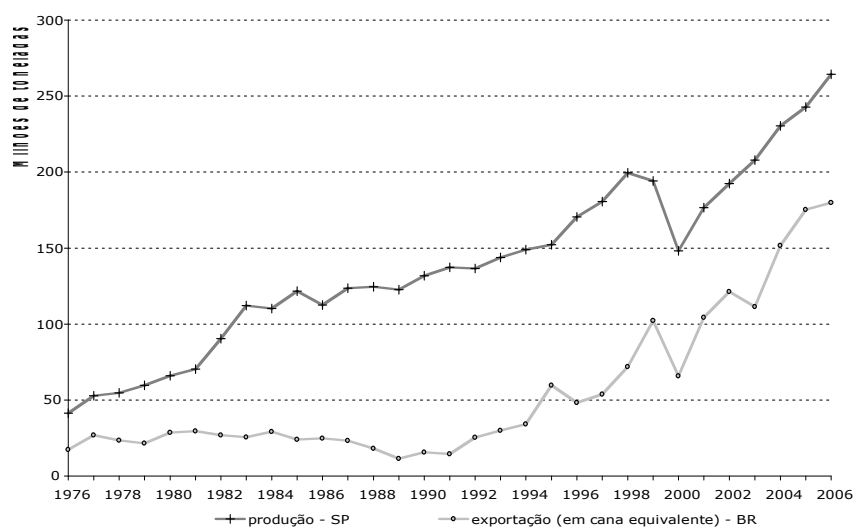

Figura 1: Séries de produção e exportação de cana-deaçúcar

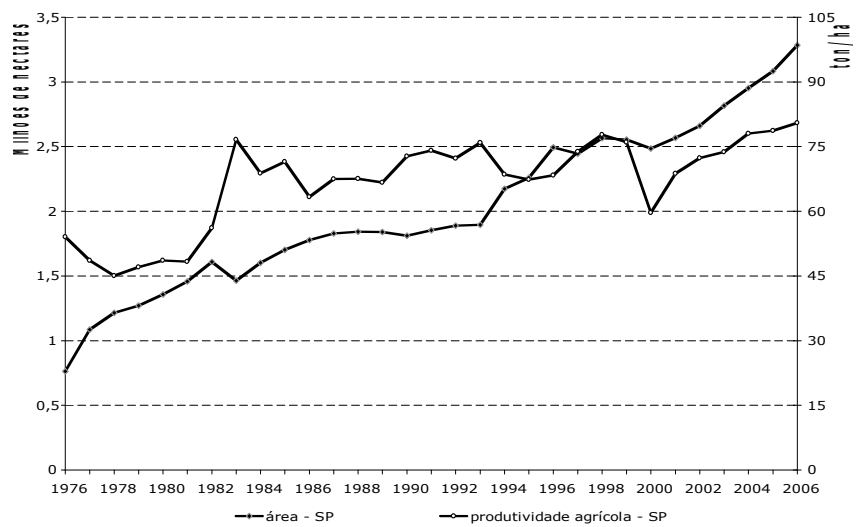

Figura 2: Séries de área plantada e produtividade agrícola da cana-de-açúcar

de demanda: preço de açúcar e álcool $\left(p^{d}\right)$ e renda $(m)$ domésticos - além da terceira variável que origina choques de oferta: o preço da cana $\left(p^{s}\right)$.

Deve-se ter em vista que os resultados aqui apresentados estão ligados apenas às variáveis supracitadas. Outras variáveis cujos choques não se encontram entre os que foram avaliados - mas que possam eventualmente afetar as variáveis de interesse - estão incluídas no resíduo do modelo estimado (o que permite que sua influência sobre as séries analisadas também seja levada em consideração).

Os resultados dos testes de raiz unitária são apresentados na Tabela 2. Em nenhuma das três formas adotadas para a pré-filtragem das séries (a saber: constante e tendência, apenas a constante e sem a inclusão de termos determinísticos) as estatísticas foram significativas ao nível de $1 \%$ de probabilidade e, por isso, todas as séries foram tomadas como I(1). Logo, o modelo foi ajustado com as variáveis nas primeiras diferenças.

O teste de Johansen indicou a existência de dois vetores de cointegração, de forma que o modelo ajustado foi um VEC - Modelo de Auto-Regressão Vetorial com Correção de Erro (Tabela 3). Os vetores de cointegração, incluídos no modelo estimado, encontram-se na Tabela 4 . O teste $\chi^{2}$ indicou que as 


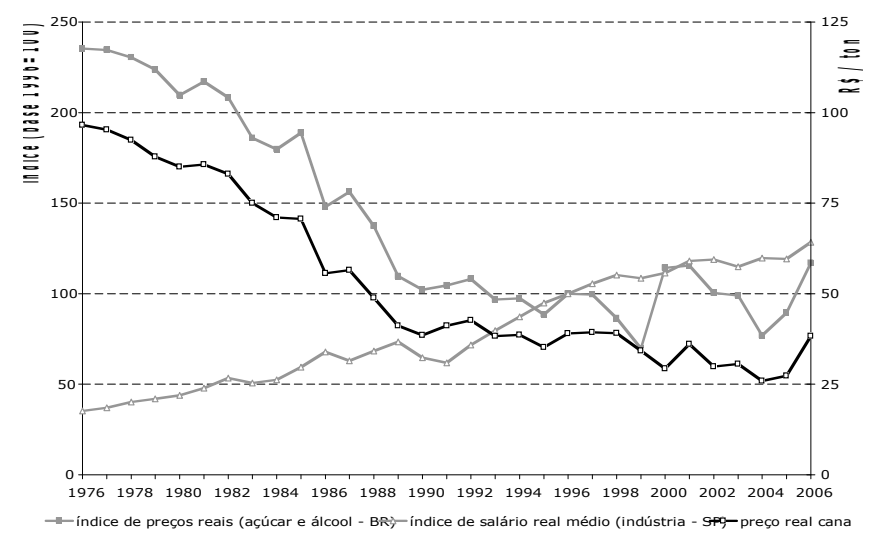

Figura 3: Séries de renda e preços domésticos

Tabela 2: Resultados dos testes de raiz unitária (DF-GLS)

\begin{tabular}{llccccc}
\hline & \multicolumn{2}{c}{ Constante e tendência } & \multicolumn{2}{c}{ Constante } & \multicolumn{2}{c}{ Sem termos determinísticos } \\
& Estatística & Defasagens & Estatística & Defasagens & Estatística & Defasagens $^{*}$ \\
\hline$n$ & $-1,327$ & 6 & 1,479 & 8 & 1,494 & 5 \\
$\theta$ & $-2,551$ & 0 & $-1,365$ & 0 & $-1,365$ & 0 \\
$p^{d}$ & $-1,978$ & 0 & $-1,15$ & 0 & $-1,15$ & 0 \\
$x$ & $-1,31$ & 1 & 0,028 & 0 & 0,028 & 0 \\
$p^{s}$ & $-1,821$ & 0 & $-0,807$ & 0 & $-0,807$ & 0 \\
$m$ & $-2,193$ & 0 & 0,233 & 0 & 0,233 & 0 \\
\hline
\end{tabular}

* O número de defasagens foi determinado de acordo com o MAIC.

Tabela 3: Resultados do testes de cointegração

\begin{tabular}{cccc}
\hline$H_{0}$ & $H_{A}$ & $\lambda_{\text {traço }}$ & Valor-P \\
\hline$h=0$ & $h>0$ & $151,132^{*}$ & 0,000 \\
$h \leq 1$ & $h>1$ & $80,749^{* *}$ & 0,024 \\
$h \leq 2$ & $h>2$ & 47,045 & 0,184 \\
$h \leq 3$ & $h>3$ & 26,401 & 0,324 \\
$h \leq 4$ & $h>4$ & 11,433 & 0,509 \\
$h \leq 5$ & $h>5$ & 4,117 & 0,407 \\
\hline${ }^{*}$ Significativo ao nível de probabilidade \\
de 1\%. \\
Significativo ao nível de probabilidade \\
de 5\%.
\end{tabular}

constantes dentro dos vetores de cointegração eram significativas e, por isso, as mesmas foram mantidas. O modelo utilizado nos testes de cointegração incluía também uma tendência linear e ele foi ajustado com uma defasagem. Essa especificação, que apresentou o melhor ajustamento entre as testadas, segundo os critérios de informação - AIC e Schwarz na versão multi-equacional, também foi utilizada para a estimativa do VEC, cujos resultados são apresentados na Tabela 5 .

Com base nos resultados dos testes econométricos apresentados, o sistema estimado foi um VEC 1 com dois vetores de cointegração. 
Tabela 4: Parâmetros estimados para os vetores de cointegração

\begin{tabular}{crr}
\hline Séries & \multicolumn{1}{c}{$h 1$} & \multicolumn{1}{c}{$h 2$} \\
\hline$n$ & 7,861 & 5,279 \\
$\theta$ & $-1,457$ & $-2,395$ \\
$p^{s}$ & 0,808 & 1,186 \\
$p^{d}$ & 0,274 & 3,100 \\
$x$ & $-0,831$ & $-1,071$ \\
$m$ & $-2,669$ & 1,538 \\
const. $\left(\alpha_{1}\right)$ & $-86,705$ & $-73,323$ \\
\hline
\end{tabular}

Conforme pode ser observado na Tabela 5, exceto pelo coeficiente estimado para a influência contemporânea do nível de preços de açúcar e álcool $\left(p^{d}\right)$ sobre a exportação do setor sucroalcooleiro $(x)$, os coeficientes apresentaram os sinais esperados. Embora o teste $t$ não seja aplicável com o mesmo rigor que em modelos uniequacionais às estimativas da metodologia VEC, essas estatísticas foram apresentadas para se ter uma idéia da precisão com que os coeficientes foram obtidos. Deve-se destacar a influência do preço da cana sobre a produtividade e do preço de açúcar e álcool sobre as exportações, cujas estatísticas $t$ (em valor absoluto) foram maiores e iguais a 2, respectivamente. Não se pode descartar, também, a influência da renda doméstica sobre as exportações.

Apesar da relação negativa entre os preços de açúcar e álcool e as exportações não estar em concordância com o modelo econômico proposto - ver eq. (13) - o resultado obtido não é completamente vazio de significado econômico. Ao contrário: de acordo com o modelo proposto, as exportações são determinadas pelo excesso de oferta no mercado interno e, por isso, o nível doméstico de preços pode ser visto como um indicador de excesso/escassez do produto no mercado interno. Ou seja, um aumento no preço do açúcar e/ou do álcool pode representar também uma escassez relativa (i.e., uma diminuição do excedente exportável) desses produtos - implicando uma relação inversamente proporcional entre a exportação e o nível doméstico de preços.

A decomposição histórica das variâncias dos erros de previsão, 10 períodos à frente, para as cinco variáveis diretamente relacionadas ao setor sucroalcooleiro - a saber: área $(n)$, produtividade $(\theta)$, exportação em cana equivalente $(x)$, preço da cana $\left(p^{s}\right)$ e índice de preço médio de açúcar e álcool $\left(p^{d}\right)$ - são apresentadas na Tabela 6. Os resultados corroboram os pressupostos de exogeneidade da área, que teve mais de $93 \%$ da variância do erro previsão sendo explicados pela própria série, e de endogeneidade das demais variáveis.

A produtividade foi responsável por $53,32 \%$ da variação da própria série, sendo o preço da cana (com $23,62 \%$ ) a segunda variável mais importante para explicá-la. Cerca de $60,52 \%$ da variação da série de preço da cana foram explicados pela própria variável e cerca de $28,13 \%$, pelos preços de açúcar e de álcool. É interessante observar que, durante o período analisado, o preço médio dos produtos finais foi mais importante para explicar a variação do preço da matéria-prima do que vice-versa: o preço da cana foi responsável por apenas $13,68 \%$ da variação do preço médio de açúcar e álcool (com essa última série sendo responsável por $70,9 \%$ da própria variação).

Entre as séries analisadas, a de exportação foi a mais endógena: apenas 
Tabela 5: Coeficientes estimados para a matriz de relações contemporâneas

\begin{tabular}{|c|c|c|c|}
\hline \multicolumn{2}{|c|}{$\begin{array}{l}\text { Influência } \\
\text { de sobre }\end{array}$} & $\begin{array}{l}\text { Coeficiente } \\
\text { estimado }\end{array}$ & Estatística $t$ \\
\hline$p^{s}$ & $\theta$ & 0,318 & 2,589 \\
\hline$p^{d}$ & $p^{s}$ & 0,214 & 0,015 \\
\hline$p^{s}$ & $p^{d}$ & 0,273 & 0,011 \\
\hline$n$ & $x$ & 0,612 & 0,376 \\
\hline$\theta$ & $x$ & 0,298 & 0,411 \\
\hline$p^{s}$ & $x$ & 0,558 & 0,775 \\
\hline$p^{d}$ & $x$ & $-0,841$ & $-2,000$ \\
\hline$m$ & $x$ & $-1,091$ & $-1,100$ \\
\hline
\end{tabular}

${ }^{a}$ Nota: Os sinais dos coeficientes já se
encontram na forma em que devem ser
analisados.

Tabela 6: Decomposição da variância dos erros de previsão

\begin{tabular}{|c|c|c|c|c|c|c|c|c|c|c|c|}
\hline Variável & Inovação & 1 & 2 & 3 & 4 & 5 & 6 & 7 & 8 & 9 & 10 \\
\hline \multirow[t]{6}{*}{$n$} & $n$ & 100,0 & 96,0 & 94,7 & 93,8 & 93,7 & 93,7 & 93,6 & 93,6 & 93,6 & 93,6 \\
\hline & $\theta$ & 0,0 & 0,8 & 0,8 & 0,9 & 0,9 & 0,9 & 0,9 & 0,9 & 0,9 & 0,9 \\
\hline & $p^{d}$ & 0,0 & 1,9 & 1,9 & 2,6 & 2,6 & 2,6 & 2,6 & 2,6 & 2,6 & 2,6 \\
\hline & $x$ & 0,0 & 0,0 & 0,0 & 0,1 & 0,1 & 0,2 & 0,2 & 0,2 & 0,2 & 0,2 \\
\hline & $m$ & 0,0 & 0,1 & 0,4 & 0,4 & 0,4 & 0,4 & 0,4 & 0,4 & 0,4 & 0,4 \\
\hline & $p^{s}$ & 0,0 & 1,1 & 2,1 & 2,2 & 2,2 & 2,2 & 2,2 & 2,2 & 2,2 & 2,2 \\
\hline \multirow[t]{6}{*}{$\theta$} & $n$ & 0,0 & 2,9 & 3,3 & 3,4 & 3,4 & 3,4 & 3,4 & 3,4 & 3,4 & 3,4 \\
\hline & $\theta$ & 81,2 & 63,7 & 57,3 & 54,2 & 53,5 & 53,4 & 53,4 & 53,4 & 53,3 & 53,3 \\
\hline & $p^{d}$ & 1,4 & 3,5 & 3,1 & 5,7 & 6,8 & 7,0 & 7,0 & 7,0 & 7,0 & 7,0 \\
\hline & $x$ & 0,0 & 11,1 & 11,2 & 11,0 & 10,8 & 10,8 & 10,9 & 10,9 & 10,9 & 10,9 \\
\hline & $m$ & 0,0 & 0,1 & 1,5 & 1,7 & 1,7 & 1,7 & 1,7 & 1,7 & 1,7 & 1,7 \\
\hline & $p^{s}$ & 17,4 & 18,7 & 23,7 & 24,0 & 23,7 & 23,6 & 23,6 & 23,6 & 23,6 & 23,6 \\
\hline \multirow[t]{6}{*}{$x$} & $n$ & 1,0 & 1,4 & 2,0 & 2,6 & 2,7 & 2,8 & 2,8 & 2,8 & 2,8 & 2,8 \\
\hline & $\theta$ & 0,7 & 0,5 & 0,5 & 1,4 & 1,8 & 1,9 & 2,0 & 2,0 & 2,0 & 2,0 \\
\hline & $p^{d}$ & 14,5 & 22,6 & 19,0 & 19,3 & 19,6 & 20,0 & 20,1 & 20,1 & 20,1 & 20,1 \\
\hline & $x$ & 73,9 & 52,9 & 49,4 & 47,2 & 46,1 & 45,7 & 45,6 & 45,6 & 45,6 & 45,6 \\
\hline & $m$ & 6,9 & 11,9 & 13,2 & 12,5 & 12,3 & 12,2 & 12,2 & 12,2 & 12,2 & 12,2 \\
\hline & $p^{s}$ & 3,0 & 10,7 & 15,9 & 17,1 & 17,4 & 17,4 & 17,4 & 17,4 & 17,4 & 17,4 \\
\hline \multirow[t]{6}{*}{$p^{s}$} & $n$ & 0,0 & 1,3 & 2,0 & 2,0 & 2,0 & 2,0 & 2,0 & 2,0 & 2,0 & 2,0 \\
\hline & $\theta$ & 0,0 & 1,3 & 3,8 & 4,2 & 4,2 & 4,2 & 4,2 & 4,2 & 4,2 & 4,2 \\
\hline & $p^{d}$ & 7,6 & 22,0 & 27,4 & 28,2 & 28,3 & 28,2 & 28,1 & 28,1 & 28,1 & 28,1 \\
\hline & $x$ & 0,0 & 3,3 & 3,3 & 3,3 & 3,6 & 3,7 & 3,8 & 3,8 & 3,8 & 3,8 \\
\hline & $m$ & 0,0 & 1,3 & 1,1 & 1,1 & 1,2 & 1,2 & 1,3 & 1,3 & 1,3 & 1,3 \\
\hline & $p^{S}$ & 92,4 & 70,8 & 62,4 & 61,1 & 60,8 & 60,6 & 60,6 & 60,5 & 60,5 & 60,5 \\
\hline \multirow[t]{6}{*}{$p^{d}$} & $n$ & 0,0 & 0,0 & 0,8 & 1,1 & 1,1 & 1,2 & 1,2 & 1,2 & 1,2 & 1,2 \\
\hline & $\theta$ & 0,0 & 1,9 & 2,3 & 3,0 & 3,1 & 3,1 & 3,2 & 3,1 & 3,2 & 3,2 \\
\hline & $p^{d}$ & 96,0 & 78,6 & 73,3 & 71,2 & 70,9 & 71,0 & 70,9 & 70,9 & 70,9 & 70,9 \\
\hline & $x$ & 0,0 & 2,7 & 5,9 & 6,2 & 6,2 & 6,2 & 6,2 & 6,2 & 6,2 & 6,2 \\
\hline & $m$ & 0,0 & 5,0 & 5,0 & 4,9 & 4,9 & 4,9 & 4,9 & 4,9 & 4,9 & 4,9 \\
\hline & $p^{s}$ & 4,0 & 11,7 & 12,8 & 13,5 & 13,7 & 13,7 & 13,7 & 13,7 & 13,7 & 13,7 \\
\hline
\end{tabular}

$45,62 \%$ de sua variação foram explicadas pela própria série. A segunda variável mais importante para explicar a variação do erro de previsão da exportação foi o preço médio de açúcar e álcool no mercado doméstico (20,08\%), seguida pelo preço da cana $(17,42 \%)$ e renda doméstica $(12,16 \%)$. Variações de área e produtividade não apresentaram um elevado poder explicativo sobre variações da exportação do setor sucroalcooleiro: $2,76 \%$ e 1,96\%, respectivamente.

As elasticidades-impulso reflexivas - ou seja, as respostas de cada variá- 


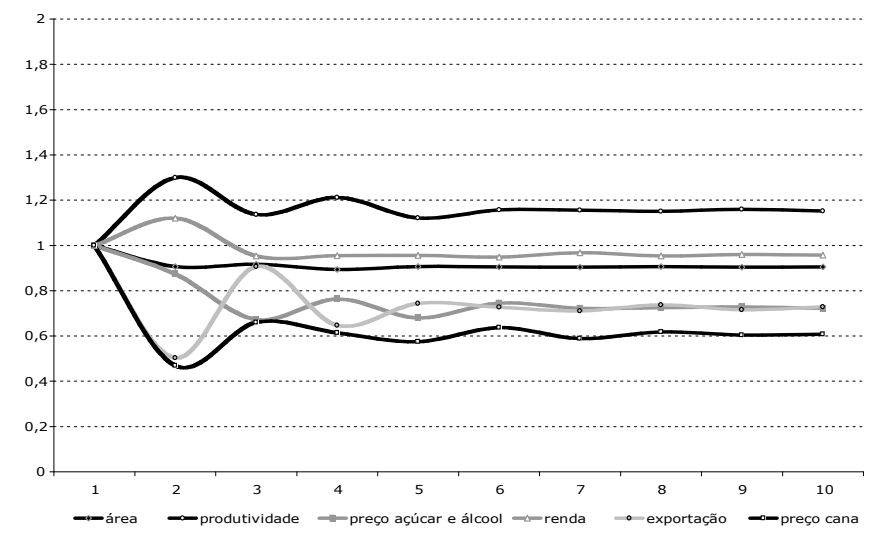

Figura 4: Elasticidade-impulso reflexiva acumulada

vel do sistema a choques ${ }^{4}$ ocorridos na própria variável- são apresentadas na Figura 4. De maneira geral, observa-se que os efeitos de choques reflexivos no setor sucroalcooleiro tenderam a se dissipar rapidamente, com as variáveis convergindo para um novo patamar de crescimento passados apenas 3 anos da variação inesperada. A produtividade agrícola foi a única variável que apresentou uma resposta mais que proporcional ao efeito do choque: por exemplo, o impacto de um aumento inesperado de $1 \%$ no crescimento da produtividade seria sentido também nas safras seguintes, atingindo o pico de 1,3\% após um ano e convergindo para 1,15\% no longo-prazo. Uma possível explicação para esse comportamento da produtividade são os ganhos associados à curva de aprendizagem: em um primeiro momento, a adoção de variedades mais adaptadas ao clima ou de novas técnicas de cultivo têm um impacto imediato sobre o rendimento da lavoura; entretanto, esses ganhos são maximizados apenas com o passar do tempo, após o domínio das novas práticas.

A Figura 5 ilustra a resposta da taxa de crescimento da área a variações inesperadas na taxa de crescimento das demais variáveis que fazem parte do modelo estimado. Como se pode observar, a área cultivada com canade-açúcar no Estado de São Paulo foi praticamente insensível a choques na produtividade, no preço médio de açúcar e álcool, na renda, na exportação e no preço da cana. Entretanto, observou-se um crescimento expressivo da área durante todo o período analisado. Esse comportamento foi possivelmente motivado pela manutenção de uma rentabilidade relativamente elevada para essa atividade agrícola durante todo o período em que esteve regulamentada.

Diferentemente do que se observou no caso da área, a taxa de crescimento da produtividade apresentou uma resposta geralmente positiva a choques nas demais variáveis (ver Figura 6). As elasticidades-impulso acumuladas para choques na exportação, na renda, no preço de médio açúcar e álcool, na área e no preço da cana sobre a área variaram entre 0,11 e 0,25 - indicando que a taxa de crescimento da produtividade foi inelástica a choques nessas variáveis. Choques positivos de demanda (sejam através de aumentos no preço médio de açúcar e álcool, na renda doméstica ou nas exportações) tendem a aumentar a taxa de crescimento do preço da cana recebido pelos produtores - estimulando o uso de técnicas mais dispendiosas que proporcionam ganhos

\footnotetext{
4 Como o modelo foi estimado nas diferenças, a interpretação dos resultados deve ser feita considerando-se que os choques são variações inesperadas na taxa de crescimento (ou redução).
} 


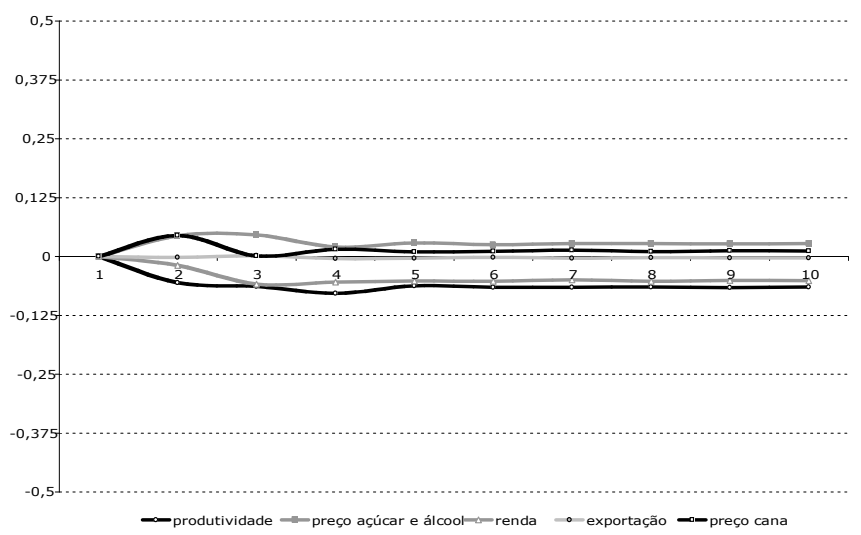

Figura 5: Elasticidade-impulso acumulada das demais variáveis sobre a área

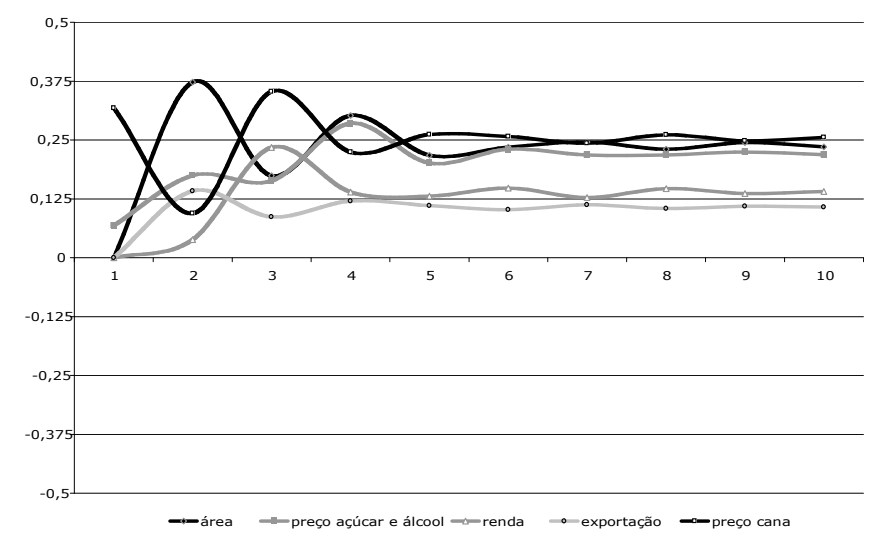

Figura 6: Elasticidade-impulso acumulada das demais variáveis sobre a produtividade

de produtividade.

Como se sabe, a cana de $1^{\circ}$ corte (ou seja, a cana plantada no ano anterior e que foi colhida pela primeira vez) apresenta uma produtividade maior do que a observada em cortes mais velhos. Essa pode ser a razão pela qual um choque de $1 \%$ na área aumentou a taxa de crescimento da produtividade agrícola média do período seguinte em $0,37 \%$.

Por outro lado, um choque de $1 \%$ no preço da cana provocou um aumento contemporâneo de $0,31 \%$ na taxa de crescimento da produtividade - mas que tende a ser reduzido já no período seguinte. Esse resultado pode estar ligado ao fato de que um aumento nos preços tende a intensificar os tratos culturais.

Na Figura 7 estão representadas as elasticidades-impulso acumuladas de choques na área, na produtividade, na exportação, na renda e no preço médio de açúcar e álcool sobre o preço da cana. Observa-se que a variável que apresentou o maior impacto sobre o preço da matéria-prima foi o preço médio dos produtos finais, cuja elasticidade acumulada foi de $0,4 \%$.

Os preços praticados no setor sucroalcooleiro foram, durante muitos anos, administrados pelo Governo. Por esse motivo, provavelmente, os preços se mostraram exógenos a variações na área, na produtividade, na exportação e na 


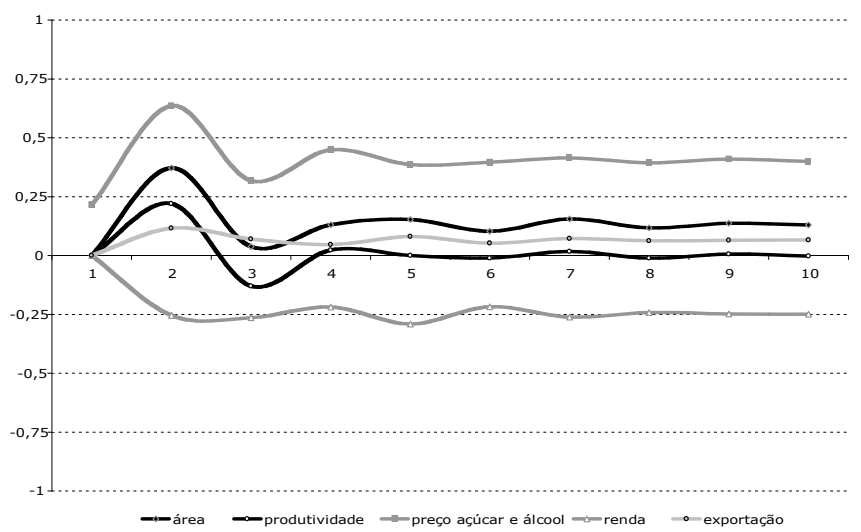

Figura 7: Elasticidade-impulso acumulada das demais variáveis sobre o preço da cana-de-açúcar

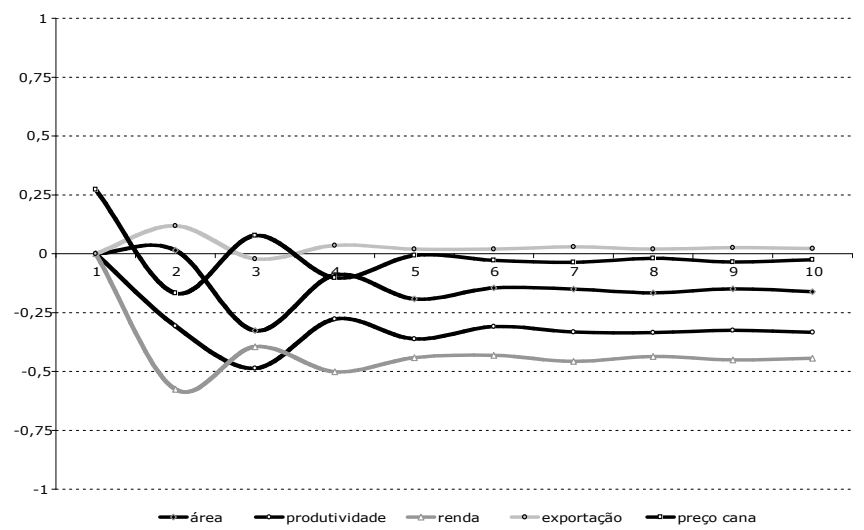

Figura 8: Elasticidade-impulso acumulada das demais variáveis sobre o preço médio de açúcar e álcool

renda doméstica. Contudo, para garantir o desenvolvimento do setor, os preços eram fixados em níveis remuneradores tanto para os produtores de cana quanto para os produtores de açúcar e álcool. Logo, havia uma relação contemporânea entre o nível de preços dos produtos finais e da matéria-prima, a qual não pode ser ignorada.

Essa relação também pode ser observada, no sentido oposto, através da Figura 8. Um aumento de $1 \%$ no crescimento do preço da cana provocou, em média, um aumento contemporâneo de $0,27 \%$ no crescimento do preço médio de açúcar e álcool. Choques de exportação também não apresentaram impactos significativos sobre o preço médio de açúcar e álcool no longo-prazo. Nesse caso, os choques de área e de produtividade apresentaram o impacto esperado sobre o nível de preços: as elasticidades impulso acumuladas são, respectivamente, $-0,16$ e - $-0,33$. Assim como sobre o preço da cana, o choque de renda apresentou um impacto negativo sobre o preço de açúcar e álcool: um aumento de $1 \%$ no crescimento da renda desaceleraria em $0,45 \%$ a taxa de crescimento do preço médio dos produtos.

Esse último resultado tem algumas possíveis explicações. Pode ser que os aumentos de renda verificados coincidam com uma diminuição do preço 


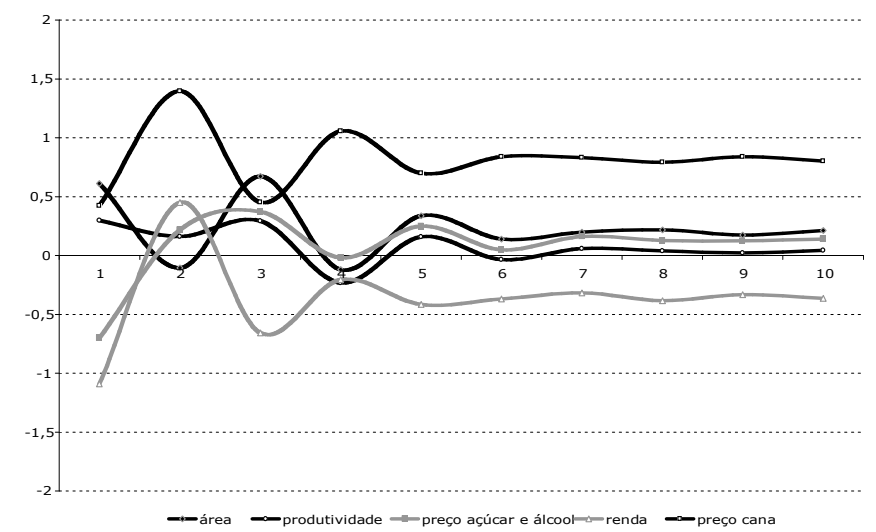

Figura 9: Elasticidade-impulso acumulada das demais variáveis sobre a exportação

médio de açúcar e álcool decorrente da liberalização do setor e dos ganhos em produtividade. Assim, variações positivas de renda podem estar relacionadas a variações negativas de preços provocadas por mudanças conjunturais do mercado e, nesse caso, não se pode inferir sobre relações de dependência entre essas variáveis.

Além disso, não se pode esquecer que, na falta de uma outra variável que representasse de forma adequada a renda média no Estado de São Paulo, a mesma foi representada pelo salário industrial médio na estimativa do modelo. Portanto, pode ser que essa variável, por se referir a uma categoria específica, não seja uma boa proxy.

Como se pode observar na Figura 9, a exportação foi a variável que apresentou maior sensibilidade a choques nas demais variáveis. De acordo com os resultados estimados, a taxa de crescimento da quantidade exportada de açúcar e álcool, em cana equivalente, foi elástica a variações contemporâneas na taxa de crescimento da renda e a variações na taxa de crescimento do preço da cana no período imediatamente anterior. Por exemplo, caso ocorra um choque de renda de $1 \%$ em determinado ano, a taxa de crescimento da exportação do setor sucroalcooleiro se reduz em 1,09\% no mesmo ano - mas cresce $0,45 \%$ (em comparação ao nível inicial) no ano seguinte, convergindo para uma redução de $0,35 \%$ no longo-prazo. Já um choque positivo de $1 \%$ na taxa de crescimento do preço da cana provocaria um aumento contemporâneo de $0,42 \%$ na taxa de crescimento das exportações, atingindo um pico de $1,4 \%$ no ano seguinte e convergindo para aumento de $0,81 \%$.

Contudo, a taxa de crescimento da exportação se mostrou inelástica a choques da produtividade, do preço médio de açúcar e álcool e da área. Nos três casos, as elasticidades-impulso acumuladas foram positivas e pequenas (respectivamente: $0,04,0,13$ e 0,2 ).

Um aumento inesperado de $1 \%$ na taxa de crescimento da produtividade dos canaviais paulistas levou a um aumento contemporâneo de $0,3 \%$ na taxa de crescimento da exportação do setor sucroalcooleiro - mantendo-a nesse ritmo mais acelerado por 2 anos. Por outro lado, um choque positivo de $1 \%$ na taxa de crescimento área provocou um aumento contemporâneo de $0,61 \%$ na taxa de crescimento da exportação.

Um choque positivo no preço de açúcar e álcool, por sua vez, apresen- 


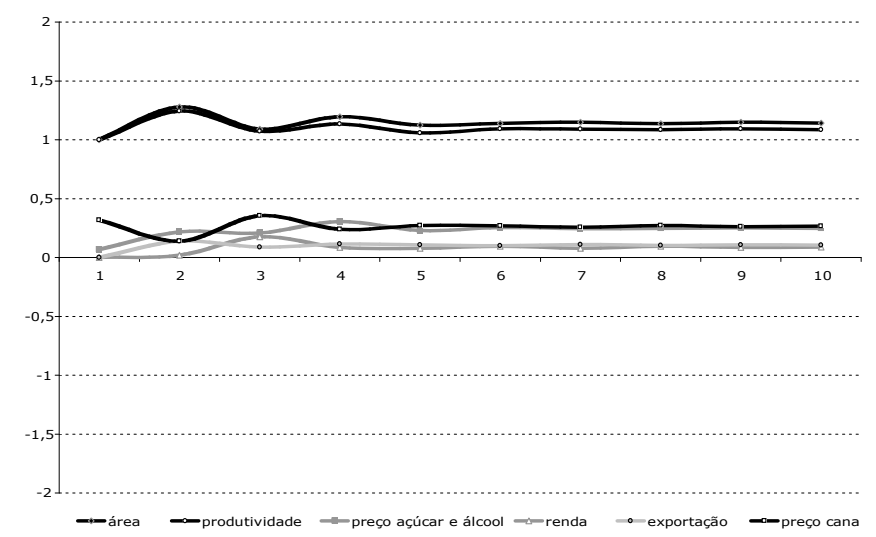

Figura 10: Elasticidade-impulso acumulada sobre a produção

tou um impacto contemporâneo negativo sobre a taxa de crescimento da exportação. Esse resultado, contrário ao que se esperava, pode ser explicado entendendo-se as variações do nível de preços médio de açúcar e álcool como um indicativo do excesso ou da escassez desses produtos no mercado doméstico. Sob essa ótica, choques positivos indicariam que a população estaria encontrando dificuldades para ter acesso aos produtos do setor sucroalcooleiro - que poderiam ter suas exportações reduzidas para atender ao mercado doméstico e controlar o nível de preços.

Todos os resultados apresentados até agora são de suma importância para uma melhor compreensão da dinâmica econômica em que o setor sucroalcooleiro esteve inserido desde 1976. Contudo, busca-se neste trabalho explicar a evolução da produção de cana-de-açúcar no Estado de São Paulo através de choques de oferta e demanda. Essa representação não pôde ser observada diretamente através do modelo proposto. Já que a equação (2) - que representa a oferta (ou seja, a produção) de cana - é um modelo aditivo cujas variáveis fazem parte do modelo estimado, o impacto de choques das outras variáveis sobre a produção pode ser obtido somando-se o impacto de tais choques sobre a área e sobre a produtividade. Para permitir uma melhor visualização, essas combinações de resultados são ilustradas na Figura 10.

No curto-prazo, a resposta da produção a choques de oferta - sejam eles oriundos da variação da área ou da produtividade - foi bastante semelhante. Um aumento inesperado de $1 \%$ na taxa de crescimento da área ou da produtividade, por razões óbvias, aumentou a taxa de crescimento contemporânea da produção em $1 \%$. No ano seguinte, a taxa de crescimento da produção aumentou ainda mais - sendo, em média, 1,26\% maior que a observada antes da inovação.

No longo-prazo, entretanto, padrões distintos puderam ser observados. Choques de oferta (área e produtividade) tiveram impactos mais que proporcionais sobre a produção, com elasticidades-impulso acumuladas de 1,14 e 1,09 , respectivamente. Por outro lado, choques de preço, tanto da própria cana quanto do açúcar e do álcool, apresentaram impactos menos que proporcionais sobre a produção, com elasticidades-impulso acumuladas convergindo para 0,25 (em ambos os casos). A produção também se mostrou inelástica a choques de demanda (renda doméstica e exportação) - mas, nesse caso, 
as elasticidades impulso acumuladas foram ainda menores (respectivamente: $0,09$ e 0,10$)$.

A resposta da produção a choques de preço, no curto-prazo, dependeu de sua origem: um aumento inesperado de $1 \%$ na taxa de crescimento do preço da cana aumentou em $0,32 \%$ a taxa de crescimento contemporânea da produção, enquanto o mesmo aumento de $1 \%$ na taxa de crescimento do preço de açúcar e álcool a aumentaria em apenas $0,07 \%$. Embora a relação contemporânea entre o preço dos produtos finais e da produção não tenha sido explicitada na matriz de relações contemporâneas, essa relação existe - ainda que de forma indireta: a produção é afetada contemporaneamente por choques no preço da cana, o qual é afetado por choques no preço de açúcar e álcool ocorridos no mesmo período. Assim, via preço da matéria-prima, choques no preço dos produtos finais afetam a produção.

Com relação aos choques de demanda, a resposta da produção no curtoprazo também diferiu de uma variável para outra. O impacto de um choque de $1 \%$ na exportação sobre a produção atingiu o pico 1 ano após sua ocorrência, aumentando em $0,14 \%$ a taxa de crescimento da produção. Já o impacto de um choque $1 \%$ na renda doméstica sobre a exportação foi sentido apenas 2 anos após sua ocorrência, quando aumenta a taxa de crescimento da produção em $0,17 \%$.

\section{Conclusões}

A análise da dinâmica econômica observada no setor sucroalcooleiro entre 1976 e 2006 evidenciou que a evolução da área cultivada com a cana-deaçúcar no Estado de São Paulo não pode ser explicada por choques de oferta (representados pela produtividade e pelo preço da cana) nem por choques de demanda (renda doméstica, preço médio de açúcar e álcool e exportação). No período em que o setor era regulamentado, o significativo crescimento da área pode ser decorrente dos níveis de preços remuneradores que foram estabelecidos pelo Estado: como os preços eram definidos tomando-se como base custos médios de produção, os produtores mais eficientes foram estimulados a expandir a produção - o que ocorre, inicialmente, através do aumento da área cultivada. Após a liberalização do setor, a continuidade desse crescimento pode ser atribuída à ocorrência de condições favoráveis nos mercados interno e externo com a expectativa de consolidação de um mercado internacional para o etanol.

Por outro lado, mais de $40 \%$ da variação histórica da produtividade se deve às outras variáveis, com destaque para o preço da cana. Já que a produção de cana-de-açúcar pode ser representada pela multiplicação da área cultivada e da produtividade da terra, as variáveis mais importantes para explicar a evolução da produção são as mais importantes para explicar a evolução da área e da produtividade. Assim, pode-se concluir que o preço da cana foi a variável de maior importância para explicar o crescimento da produção haja visto seu expressivo efeito sobre a produtividade. Os choques de oferta predominaram ao longo do período analisado, conduzindo a evolução recente da produção de cana-de-açúcar no Estado de São Paulo aos níveis atuais.

De maneira geral, os resultados estimados com o modelo econômico proposto foram satisfatórios e apresentaram os sinais esperados. A baixa magnitude estimada para as elasticidades-impulso, em muitos casos, pode ser ex- 
plicada pela intensa regulação estatal que pairou durante muitos anos sobre o setor sucroalcooleiro. Embora o processo de desregulamentação no Brasil tenha sido completado em 1999, o impacto estimado para a maior parte dos choques sobre a produtividade, sobre o preço médio de açúcar e álcool, sobre as exportações e sobre a produção foram permanentes - o que quer dizer que o efeito de choques ocorridos sobre tais variáveis no passado podem ser observáveis ainda hoje.

A constatação de que as variações do preço da cana-de-açúcar foram menos importantes para explicar as variações do preço médio de açúcar e álcool do que o contrário, pode refletir a adoção do modelo CONSECANA para precificação da matéria-prima nos últimos anos. Durante o período de intervenção estatal, o governo fixava tanto o preço da cana quanto o preço do açúcar e do álcool. Nesse contexto, esperar-se-ia que o poder de explicação recíproco fosse equivalente. Com o CONSECANA, o preço da cana é determinado com base nos preços praticados nos mercados de açúcar e álcool e, por isso, esperarse-ia uma relação causal apenas dos produtos finais para a matéria-prima. $\mathrm{O}$ resultado obtido pode ser visto como uma média de tais efeitos, ponderada pelo período em que cada conjuntura esteve vigente.

A comparação dos resultados estimados neste estudo com os obtidos por Alves et al. (2008), que analisou a produção de algodão, revela que a produção de cana-de-açúcar é menos elástica a choques de preço: enquanto um aumento não-antecipado de $1 \%$ no crescimento do preço do algodão aumentaria em $0,4 \%$ o crescimento de sua produção, no caso da cana-de-açúcar, um aumento análogo elevaria em apenas $0,25 \%$ o crescimento da produção. Isso também se repete no tocante às exportações: um choque positivo de $1 \%$ no preço do algodão aumentaria em $2 \%$ a taxa de crescimento de suas exportações, enquanto um choque positivo de $1 \%$ no preço da cana aumentaria em apenas $0,81 \%$ a taxa de crescimento das exportações de açúcar e álcool.

Uma das principais contribuições desse estudo foi a adaptação do modelo utilizado por Barros et al. (2006) e Alves et al. (2008) ao setor sucroalcooleiro, fato que permitiu avaliar o impacto de choques de oferta e de demanda sobre a produção de cana-de-açúcar no Estado de São Paulo e captar o efeito de variações da produtividade agrícola dessa cultura sobre as exportações do setor. Considerando o preço da cana-de-açúcar e o preço médio de açúcar e álcool como variáveis de ajustamento do mercado, os resultados estimados estão em linha com a proposta de Blanchard e Quah (1989): choques de demanda (renda doméstica e exportação) sobre a produção são temporários; já os choques de oferta (área e produtividade) são permanentes.

A principal limitação é o curto período das séries que, por questões relacionadas à metodologia utilizada, restringe o número de variáveis que poderiam ser utilizadas no modelo. Fica como sugestão para trabalhos futuros, quando o período pós-desregulamentação e a amostra puderem ser maiores, a inclusão de outras variáveis como o preço internacional de açúcar e álcool, o preço do petróleo e/ou até o preço da energia elétrica (neste último caso, tendo em vista as perspectivas do setor).

\section{Referências Bibliográficas}

L. R. A. Alves. Tranmissão de preços entre produtos de setor sucloalcooleiro do estado de são paulo. Master's thesis, Universidade de São Paulo - Escola 
superior de Agricultura "Luiz de Queiroz", 2002.

L. R. A. Alves. A reestruturação da cotonicultura no Brasil: fatores econômicos, institucionais e tecnológicos. PhD thesis, Universidade de São Paulo - Escola Superior de Agricultura "Luiz de Queiroz", 2006.

L. R. A. Alves, G. S. C. Barros, e M. R. P. Bacchi. Produção e expostação de algodão: efeitos de choques de oferta e de demanda. Revista Brasileira de Economia, 62:383-408, 2008.

M. R. P. Bacchi. A indústria da cana-de-açúcar: evolução e perspectivas. Technical report, BM\&FBOVESPA, 2009.

R. P. Barros, M. Carvalho, e S. Franco. Pobreza multidimensional no brasil. Technical report, 2006.

O. J. Blanchard e D. Quah. The dynamic effects of aggregate demand and supply disturbances. American Economic Review, 39:665-673, 1989.

CNA. Balanço e perspectivas da agropecuária brasileira: 2007 e 2008. Technical report, Confederação da Agricultura e Pecuária do Brasil, 2008.

C. C. da Costa. Formação de preços de açúcar e álcool combustível anidro e hidratado no estado de são paulo. Master's thesis, Universidade de São Paulo - Escola Superior de Agricultura "Luiz de Queiroz", 2000.

G. Elliot, T. J. Rothenberg, e J. H. Stock. Efficient tests for an autoregressive unit root. Technical report, NBER, 1992.

S. Johansen. Statistical analysis of cointegration vectors. Journal of Economical Dynamics and Control, 12:231-254, 1988.

H. Lütkepohl. Introduction to multiple time series analysis. Springer-Verlag, 1991.

A. Mariante. Apoio do bndes ao setor de biocombustíveis. Technical report, Banco Nacional de Desenvolvimento Econômico e Social, 2007.

M. C. Marjotta-Maistro. Ajustes nos mercados de álcool e gasolina no processo de desregulamentação. PhD thesis, Universidade de São Paulo - Escola Superior de Agricultura "Luiz de Queiroz", 2002.

T. Szmrecsányi. O planejamento da agroindústria canavieira no Brasil (19301975). Hucitec, 1979. 